\title{
JNK pathway inhibition enhances chemotherapeutic sensitivity to Adriamycin in nasopharyngeal carcinoma cells
}

\author{
YONG LIU*, JING FENG*, MING ZHAO*, JINGBO WU, JUAN FAN, \\ QINGLIAN WEN, JINHUI XU, JIANWEN ZHANG, SHAOZHI FU, BIQIONG WANG, \\ YUN LU, KANG XIONG, LI XIANG, YANLING ZHANG and LINGLIN YANG \\ Department of Oncology, The Affiliated Hospital of Southwest Medical University, \\ Luzhou, Sichuan 646000, P.R. China
}

Received October 23, 2015; Accepted March 21, 2017

DOI: $10.3892 / 01.2017 .6349$

\begin{abstract}
The role of c-Jun N-terminal kinases (JNKs) in the pathogenesis of cancer is well-known due to their involvement in carcinogenesis. Although previous studies have discussed different functions of JNKs depending on cell type, the present study aimed to investigate the function of JNKs in nasopharyngeal carcinoma (NPC) cells, as well as their involvement in chemotherapy sensitivity to Adriamycin. The present results showed that Adriamycin administration reduced cell viability and led to elevated expressions of c-Jun, phosphorylated JNK and phosphorylated c-Jun, indicating an activated JNK pathway. Notably, JNK inhibition by SP600125 also reduced cell growth. Thus, Adriamycin treatment combined with SP600125 was more effective on cell growth inhibition than each agent alone. The apoptosis analysis confirmed the reduction in cell growth. Therefore, these data provide evidence that the JNK pathway activity is negatively associated with cell viability, and its decline could sensitize NPC cells to Adriamycin.
\end{abstract}

\section{Introduction}

Nasopharyngeal carcinoma (NPC) is a squamous cell carcinoma caused by a combination of factors, such as viral, dietary and genetic factors (1). It is widely diffused in Southern China and Southeast Asia and it is notorious for its local invasion and distant metastasis at the time of diagnosis (2).

Correspondence to: Dr Ming Zhao or Professor Linglin Yang, Department of Oncology, The Affiliated Hospital of Southwest Medical University, 25 Taiping Street, Luzhou, Sichuan 646000, P.R. China

E-mail: zhaoming24@126.com

E-mail: yangllluyi@126.com

*Contributed equally

Key words: c-Jun N-terminal kinase pathway, chemotherapeutic sensitivity, nasopharyngeal carcinoma, apoptosis
Chemotherapy and radiotherapy are the main strategies to treat NPC patients (3). However, poor prognosis and therapeutic failure are the most common outcomes subsequent to treatment of NPC at an advanced stage, even with concurrent chemoradiotherapy (4). Therefore, chemosensitization or radiosensitization is essential for the successful treatment of NPC.

Treatment with chemotherapeutic drugs activates several biochemical signaling pathways, including the c-Jun $\mathrm{N}$-terminal kinase (JNK) pathway (5). JNKs are serine/threonine kinases that belong to the mitogen-activated protein kinase family (6), and are responsive to diverse stress $(7,8)$. When phosphorylated by upstream kinases, JNK in turn phosphorylates and activates three nuclear transcription factors, consisting of c-Jun, activating transcription factor 2 and Elk-1 (9-11), which regulate several important cellular functions, including cell growth, differentiation, survival and apoptosis.

Previous studies have revealed the conflicting roles of JNK, depending on tumor type (12). JNK pathway inactivation decreases tumor growth in gastric or intestinal tumors $(13,14)$, indicating its pro-tumorigenic role. By contrast, JNK pathway inactivation promotes tumor development in breast cancer $(15,16)$, while JNK activation can reduce cell proliferation and maintain epithelial differentiation in non-small cell lung cancer (NSCLC) cells (17), suggesting its anti-tumorigenic role. The JNK pathway also mediates opposite functions on apoptosis in a tumor type-dependent manner, such as a pro-apoptotic role in human gastric cancer cells (18) vs. an anti-apoptotic role in SCLC cells (19).

The present study explored the role of the JNK pathway in NPC 5-8F cell line, as well as its influence on chemotherapeutic sensitivity to Adriamycin. The present results may suggest that the members of the JNK pathway could represent valid therapeutic targets on NPC treatment.

\section{Materials and methods}

Cell culture. 5-8F cells were cultured in RPMI-1640 medium (Gibco; Thermo Fisher Scientific, Inc., Waltham, MA, USA) supplemented with $10 \%$ fetal bovine serum (GE Healthcare Life 
Sciences, Chalfont, UK), $100 \mathrm{U} / \mathrm{ml}$ penicillin and $100 \mu \mathrm{g} / \mathrm{ml}$ streptomycin (Beyotime Institute of Biotechnology, Haimen, China) at $37^{\circ} \mathrm{C}$ in a $5 \% \mathrm{CO}_{2}$ humidified atmosphere.

MTT assay. Briefly, cells were seeded at a density of $0.6 \times 10^{4}$ cells per well in $100 \mu 1$ RPMI-1640 medium in a 96-well plate. Subsequent to incubation with different concentrations of Adriamycin $(0.1,0.5,1,5$ and $10 \mu \mathrm{g} / \mathrm{ml}$; Zhejiang Hisun Pharmaceutical Co. Ltd, Taizhou, China) and the JNK inhibitor SP600125 $(2.5,5,10,20$ and $40 \mu \mathrm{M}$; Beyotime Institute of Biotechnology, Beijing, China) for $24 \mathrm{~h}$, MTT assay was performed by the MTT Cell Proliferation and Cytotoxicity Assay Kit (Beyotime Institute of Biotechnology, Beijing, China). The absorbance was measured at $490 \mathrm{~nm}$ using aN iMark Spectramax microplate reader (Bio-Rad Laboratories, Inc., Hercules, CA, USA).

Western blot analysis. Total proteins from treated or untreated cells were extracted by radioimmunoprecipitation buffer with protease inhibitor (Beyotime Institute of Biotechnology), and separated by electrophoresis in SDS-PAGE gels. Next, proteins were transferred to polyvinylidene difluoride membranes (GE Healthcare Life Sciences), followed by immunoblotting with 5\% milk and incubation with the following antibodies: Phosphorylated JNK (dilution, 1:1,000; catalog no., 4668; Cell Signaling Technology, Inc., Danvers, MA, USA); c-Jun (dilution, 1:1,000; catalog no., 9165; Cell Signaling Technology, Inc.); phosphorylated c-Jun (dilution, 1:1,000; catalog no., 9164; Cell Signaling Technology, Inc.); and GAPDH (dilution 1:2,000; catalog no., 2118; Cell Signaling Technology, Inc.). The next day, membranes were washed with TBST and incubated with secondary antibodies (dilution, 1:3,000; catalog no., SA00001-2; ProteinTech, Chicago, IL, USA) for 1-2 h. Subsequent to washing with TBST, an electrochemiluminescence Luminata ${ }^{\text {Ti }}$ Crescendo Western horseradish peroxidase substrate (catalog no., WBLUR0100; EMD Millipore, Billerica, Ma, USA) was performed (GE Healthcare Life Sciences) for the detection of immunoblotting according to the protocol of the manufacturer.

TUNEL assay. Cells were seeded onto a $24-$ well plate $\left(2 \times 10^{4}\right.$ cells in $0.5 \mathrm{ml}$ medium per well), and treated with Adriamycin $(0.1 \mu \mathrm{g} / \mathrm{ml})$ in combination with SP600125 $(2.5 \mu \mathrm{M})$, as well as each agent alone. Twenty-four $\mathrm{h}$ later, TUNEL assay was performed using an In Situ Cell Death Detection kit (Roche Applied Science, Mannheim, Germany), according to the manufacturer's instructions. In total, three fields per slice were randomly selected and analyzed, and each experiment was repeated three times. The apoptotic index was calculated as the ratio of the TUNEL positive cell number to the total cell number in each field.

Flow cytometry. Cells were seeded onto 6-well plate $\left(15 \times 10^{4}\right.$ cells in $2 \mathrm{ml}$ medium per well), and treated with Adriamycin $(0.5 \mu \mathrm{g} / \mathrm{ml})$ in combination with SP600125 $(10 \mu \mathrm{M})$, as well as each agent alone. Twenty-four $\mathrm{h}$ later, cells were collected and stained for Annexin V-fluorescein isothiocyanate (FITC) and propidium iodide (PI) for $15 \mathrm{~min}$ at room temperature, according to the

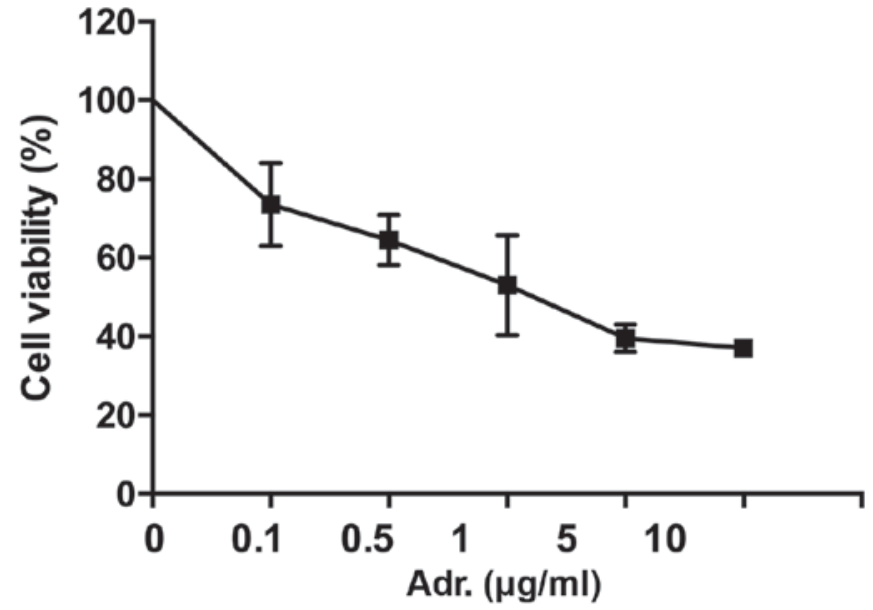

Figure 1. Adr. reduced nasopharyngeal carcinoma cell viability. 5-8F cells were treated with increasing concentrations of Adr. $(0-10 \mu \mathrm{g} / \mathrm{ml})$ for $24 \mathrm{~h}$, and then cell viability was assessed by MTT assay. Data are expressed as the mean \pm standard deviation of at least triplicate experiments. Adr., Adriamycin.

\section{$\begin{array}{lllllll}0 & 0.1 & 0.5 & 1 & 5 & 10 & \mu \mathrm{g} / \mathrm{ml}\end{array}$}

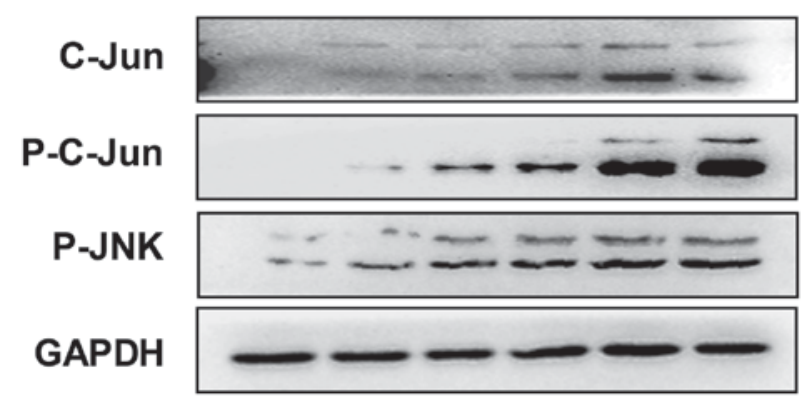

Figure 2. Adriamycin activated the JNK signaling pathway in nasopharyngeal cells. $5-8 \mathrm{~F}$ cells were treated with increasing concentrations of Adriamycin $(0-10 \mu \mathrm{g} / \mathrm{ml})$ for $24 \mathrm{~h}$, and then expression levels of members in JNK pathway were analyzed by western blot analysis. P-, phosphorylated; JNK, c-Jun N-terminal kinase.

manufacturer's instructions (Invitrogen; Thermo Fisher Scientific, Inc.). Cells were subsequently analyzed by flow cytometry (FACScan; Becton Dickinson, Franklin Lakes, NJ, USA).

Statistical analysis. Data were analyzed by SPSS 16.0 software (SPSS, Inc., Chicago, IL, USA) and GraphPad Prism 5.0 (GraphPad Software, Inc., La Jolla, CA, USA), and expressed as the mean \pm standard deviation. Statistical analysis was performed using paired Student's t-test. $\mathrm{P}<0.05$ was considered to indicate a statistically significant difference.

\section{Results}

Adriamycin caused dose-dependent cytotoxicity on NPC cells. The effect of Adriamycin on NPC cells was evaluated by MTT assay (Fig. 1). 5-8F cells were treated with different concentrations of Adriamycin. Twenty-four h later, the cell growth was inhibited, with this being statistically significant in cells treated with $\geq 0.1 \mu \mathrm{g} / \mathrm{ml}$ Adriamycin $(\mathrm{P}<0.05)$. These 

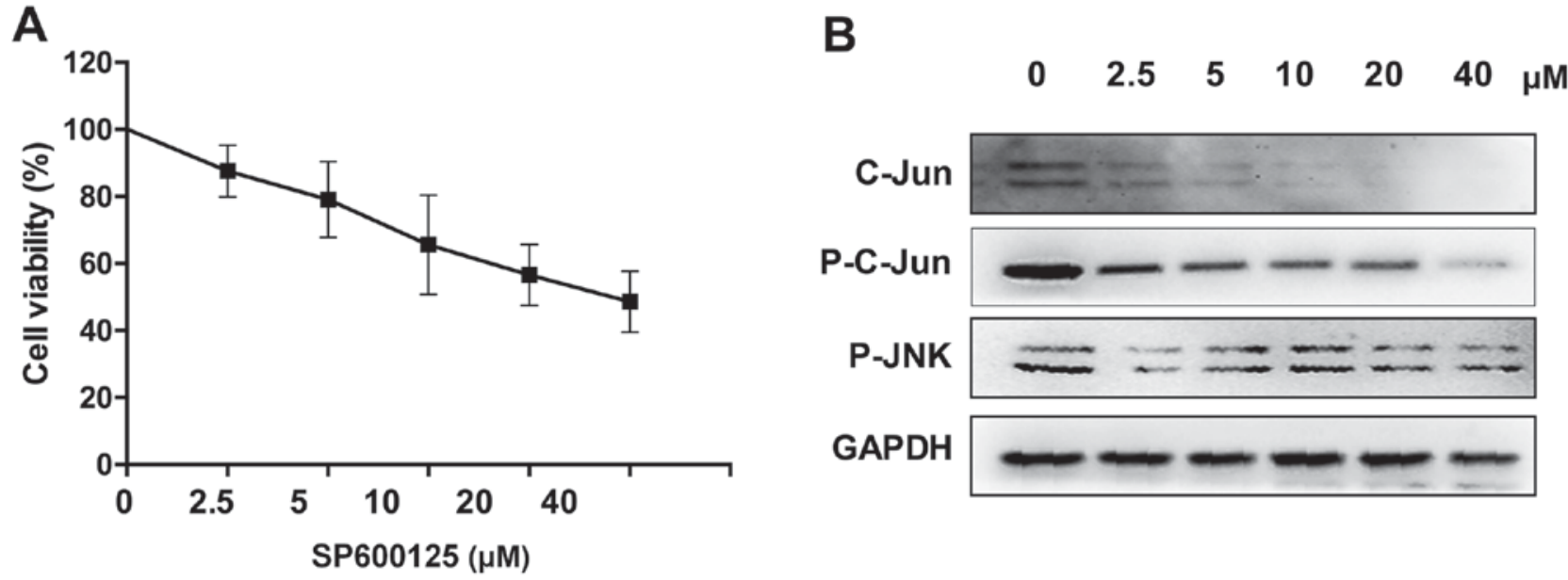

Figure 3. JNK pathway inhibition reduced NPC cells viability. 5-8F cells were treated with increasing concentrations of SP600125 (0-40 $\mu \mathrm{M})$ for 24 h. (A) Cell viability was assessed by MTT assay. Data are expressed as the mean \pm standard deviation of at least triplicate experiments. (B) Western blot analysis demonstrated high inhibition of the JNK pathway. P-, phosphorylated; JNK, c-Jun N-terminal kinase.

data indicated 5-8F cells were sensitive to Adriamycin and showed concentration-dependent sensibility.

Adriamycin activated JNK signaling pathway in NPC cells. The expression of c-Jun, phosphorylated-JNK and phosphorylated-c-Jun was analyzed to evaluate whether the JNK signaling pathway was involved in Adriamycin-associated cell inhibition in NPC cells. The present results showed an increased expression of these genes in $5-8 \mathrm{~F}$ cells treated with Adriamycin, indicating an activation of the JNK pathway (Fig. 2).

JNK signaling pathway inhibition caused cytotoxicity on NPC cells. To investigate the effect of the JNK pathway on the viability of NPC cells, 5-8F cells were treated with the JNK pathway inhibitor SP600125 to block the signaling pathway. Western blot analysis demonstrated high inhibition efficiency (Fig. 3B). Following treatment for $24 \mathrm{~h}$ at a concentration of $5 \mu \mathrm{M}$, the number of $5-8 \mathrm{~F}$ cells was $79 \%(\mathrm{P}<0.05)$, indicating that JNK signaling pathway inhibition can reduce NPC cells growth (Fig. 3A).

JNK pathway inhibition sensitized NPC cells to Adriamycin. Since the aforementioned results revealed that Adriamycin was an effective agent against NPC cells and activated the JNK pathway, which was a growth promoter in NPC cells, it was examined whether JNK pathway inhibition enhanced chemosensitivity of NPC cells to Adriamycin. In the presence of $10 \mu \mathrm{M}$ SP600125, the toxic effect of Adriamycin was evidently increased by $15 \%$ for $5-8 \mathrm{~F}$ cells $(\mathrm{P}<0.05)$ compared with Adriamycin alone (Fig. 4). These results provide an indication that JNK pathway inhibition sensitized NPC cells to the cytotoxic effects of Adriamycin.

Adriamycin combined with JNK pathway inhibition induced apoptosis in NPC cells. To investigate the effect of Adriamycin and JNK pathway inhibition on apoptotic cell death, NPC cells treated with either agent or a combination of the two were stained with Annexin V FITC/PI and TUNEL, which were analyzed by flow cytometry and fluorescent microscopy, respectively. As shown in Fig. 5, following treatment with low concentrations of Adriamycin $(0.1 \mu \mathrm{g} / \mathrm{ml})$ or SP600125 $(2.5 \mu \mathrm{M})$ alone, the cellular apoptotic rate in $5-8 \mathrm{~F}$ cells increased between 3.9 and $6.6 \%(\mathrm{P}<0.05)$, and 3.9 and $10.4 \%(\mathrm{P}<0.01)$, respectively, while the combination treatment increased between 3.9 and $14.1 \%(\mathrm{P}<0.001)$. The results were also confirmed by treatment with increased concentrations of Adriamycin $(0.5 \mu \mathrm{g} / \mathrm{ml})$ or SP600125 $(10 \mu \mathrm{M})$, which were analyzed by flow cytometry (Fig. 5C). These data demonstrated increased efficiency in the apoptosis induction by Adriamycin, combined with JNK pathway inhibition.

\section{Discussion}

Chemotherapy is the main strategy for cancer treatment, but drug resistance occurs to limit the efficacy in clinical use. Currently, the screening of agents inducing chemosensitivity is the main method to overcome this problem (20). In the present study, JNK pathway inhibition was shown to cause cytotoxicity on NPC cells, indicating that this signaling pathway exerts a growth promotion function in NPC cells. 5-8F cells treated with Adriamycin showed activated JNK signaling, which may resist Adriamycin-induced cell death. According to these findings, 5-8F cells treated with a combination of the JNK pathway inhibitor SP600125 at a fixed concentration and Adriamycin at increasing concentrations showed elevated cell death compared with cells treated with each agent alone.

In previous years, studies have revealed multiple complicated signaling networks in NPC growth inhibition $(21,22)$. The present study found that the JNK pathway was activated $24 \mathrm{~h}$ subsequent to Adriamycin administration, which was consistent with a study reporting that the JNK pathway was sensitive to cytotoxic drugs (23).

Previous studies have described the conflicting roles of the JNK pathway in tumor growth $(13,14)$. In the present study, $5-8 \mathrm{~F}$ cells were treated with a JNK pathway inhibitor, resulting in significantly decreased cell viability and increased apoptotic 

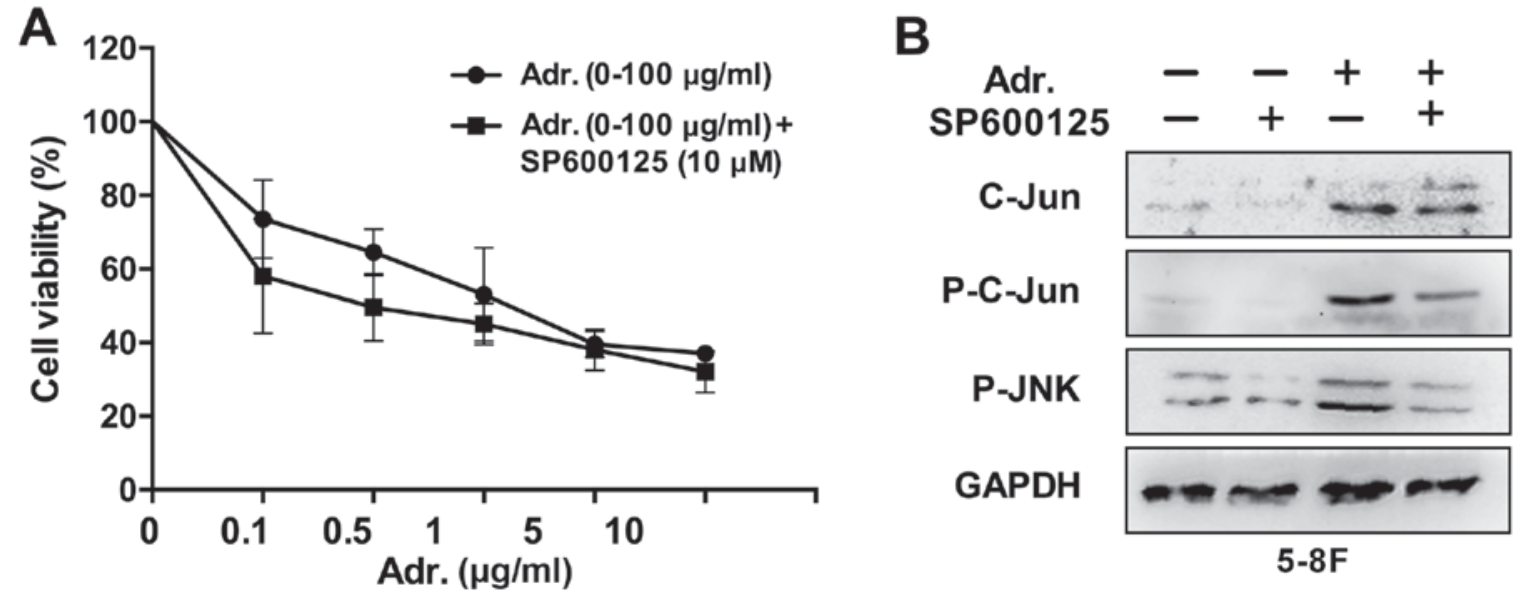

Figure 4. JNK pathway inhibition sensitized nasopharyngeal carcinoma cells to Adr. 5-8F cells were treated with $10 \mu \mathrm{M}$ SP600125 and increasing concentrations of Adr. $(0-10 \mu \mathrm{g} / \mathrm{ml})$ for $24 \mathrm{~h}$. (A) Cell viability was assessed by MTT assay. Data are expressed as the mean \pm standard deviation of at least triplicate experiments. (B) Western blot analysis demonstrated the activation of the JNK pathway. P-, phosphorylated; JNK, c-Jun N-terminal kinase; Adr., Adriamycin.

A
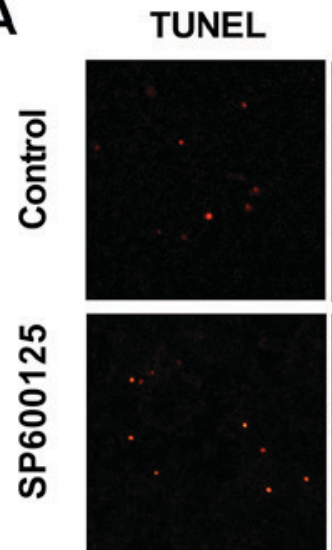

ํํㄴ

产

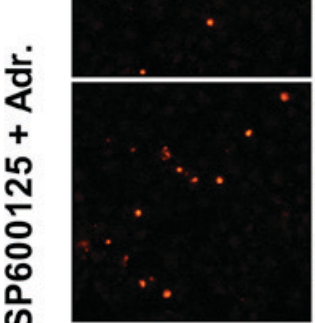

DAPI
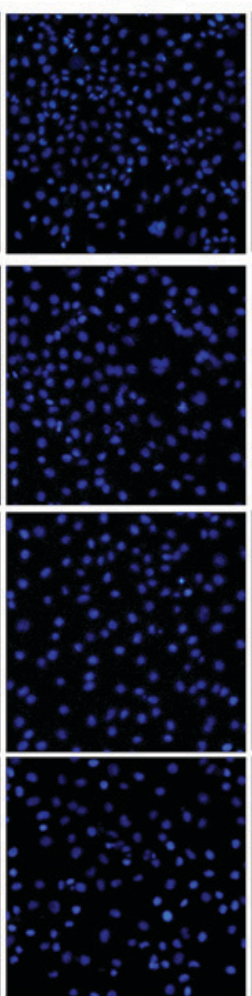

Merged
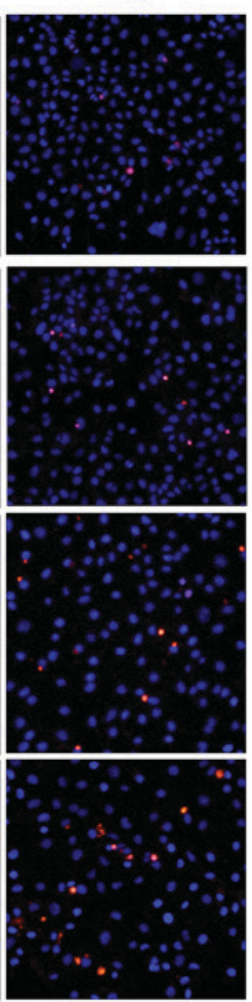

B

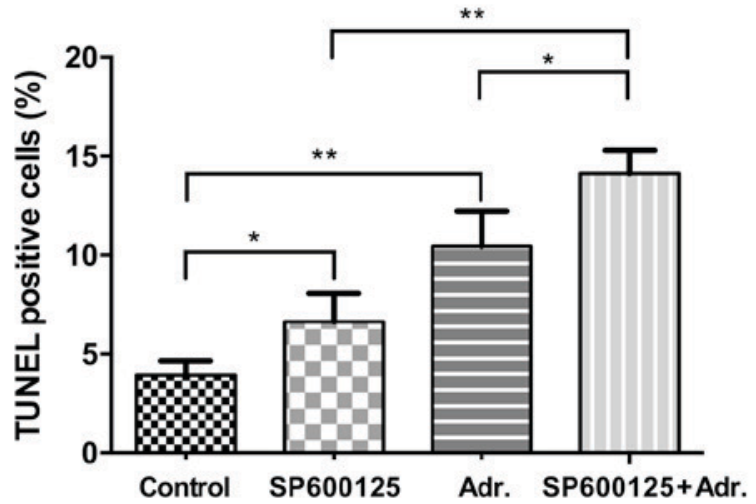

C

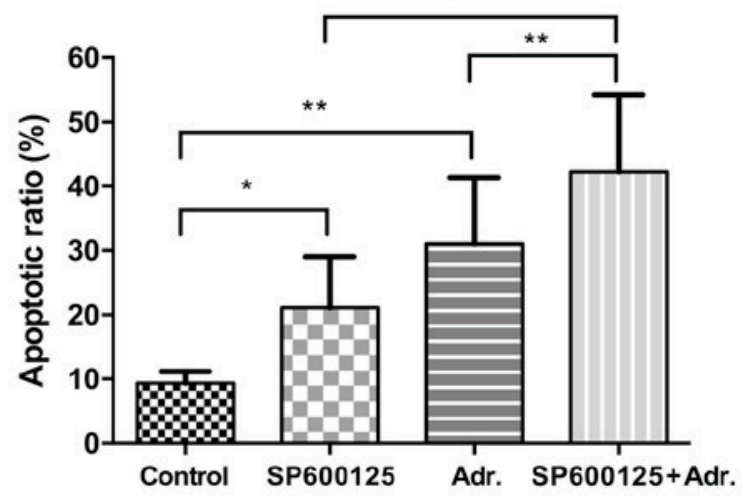

Figure 5. Adr. combined with the JNK pathway inhibitor induced apoptosis in nasopharyngeal cells. (A) TUNEL assay was used to examine the induced apoptosis at $24 \mathrm{~h}$ subsequent to treatment. Red dots represent TUNEL-positive cells, and blue dots represent nuclei staining by DAPI. The magnification was x100. (B) Apoptosis was expressed as the percentage of TUNEL-positive cells over the total number of cells. For quantification, a minimum of 3 slides and 3 fields on each slide were analyzed. (C) Quantification of apoptotic rate by flow cytometry. ${ }^{*} \mathrm{P}<0.05,{ }^{* *} \mathrm{P}<0.01$ and ${ }^{* * *} \mathrm{P}<0.01$ compared with the untreated group. P-, phosphorylated; JNK, c-Jun N-terminal kinase; Adr., Adriamycin.

rate, suggesting its pro-tumorigenic role and anti-apoptotic activity.

Overall, the present study showed that the inhibition of the JNK pathway sensitized NPC cells to the cytotoxic effects of Adriamycin, which resulted in cell growth inhibition and apoptosis. Since the function of the JNK pathway in cancer pathogenesis is dependent on cancer cell type, whether this strategy may be applied to other cancers remains unknown, and additional studies are required.

\section{Acknowledgements}

This study was supported by grants from the National Natural Science Foundation (grant no. 81201784), Scientific Research 
Foundation of the Education Department of Sichuan Province (grant no. 15ZA0163), and The Affiliated Hospital Of Southwest Medical University Foundation (grant no. 201519\#).

\section{References}

1. Zhang F and Zhang J: Clinical hereditary characteristics in nasopharyngeal carcinoma through Ye-Liang's family cluster. Chin Med J (Engl) 112: 185-187, 1999.

2. Li XJ, Ong CK, Cao Y, Xiang YQ, Shao JY, Ooi A, Peng LX, Lu WH, Zhang Z, Petillo D, et al: Serglycin is a theranostic target in nasopharyngeal carcinoma that promotes metastasis. Cancer Res 71: 3162-3172, 2011.

3. Lee AW, Lin JC and Ng WT: Current management of nasopharyngeal cancer. Semin Radiat Oncol 22: 233-244, 2012.

4. Zhang L, Chen QY, Liu H, Tang LQ and Mai HQ: Emerging treatment options for nasopharyngeal carcinoma. Drug Des Devel Ther 7: 37-52, 2013.

5. Chang PY, Wu ZZ, Sun NK and Chao CC: EBV-encoded LMP-1 sensitizes nasopharyngeal carcinoma cells to genotoxic drugs by down-regulating Cabin1 expression. J Cell Physiol 229: 309-322, 2014.

6. Johnson GL and Lapadat R: Mitogen-activated protein kinase pathways mediated by ERK, JNK, and p38 protein kinases. Science 298: 1911-1923, 2002.

7. Kyriakis JM and Avruch J: Mammalian MAPK signal transduction pathways activated by stress and inflammation: A 10-year update. Physiol Rev 92: 689-737, 2012.

8. Vlahopoulos S and Zoumpourlis VC: JNK: A key modulator of intracellular signaling. Biochemistry (Mosc) 69: 844-854, 2004.

9. Hibi M, Lin A, Smeal T, Minden A and Karin M: Identification of an oncoprotein- and UV-responsive protein kinase that binds and potentiates the c-Jun activation domain. Genes Dev 7: 2135-2148, 1993.

10. Gupta S, Campbell D, Dérijard B and Davis RJ: Transcription factor ATF2 regulation by the JNK signal transduction pathway. Science 267: 389-393, 1995.

11. Cavigelli M, Dolfi F, Claret FX and Karin M: Induction of c-fos expression through JNK-mediated TCF/Elk-1 phosphorylation. EMBO J 14: 5957-5964, 1995.

12. Tournier C: The 2 Faces of JNK Signaling in Cancer. Genes Cancer 4: 397-400, 2013.

13. Shibata W, Maeda S,Hikiba Y, Yanai A, Sakamoto K, Nakagawa H, Ogura K, Karin M and Omata M: c-Jun NH2-terminal kinase 1 is a critical regulator for the development of gastric cancer in mice. Cancer Res 68: 5031-5039, 2008.
14. Nateri AS, Spencer-Dene B and Behrens A: Interaction of phosphorylated c-Jun with TCF4 regulates intestinal cancer development. Nature 437: 281-285, 2005.

15. Cellurale C, Weston CR, Reilly J, Garlick DS, Jerry DJ, Sluss HK and Davis RJ: Role of JNK in a Trp53-dependent mouse model of breast cancer. PLoS One 5: e12469, 2010.

16. Cellurale C, Girnius N, Jiang F, Cavanagh-Kyros J, Lu S, Garlick DS, Mercurio AM and Davis RJ: Role of JNK in mammary gland development and breast cancer. Cancer Res 72: 472-481, 2012.

17. Winn RA, Marek L, Han SY, Rodriguez K, Rodriguez N, Hammond M, Van Scoyk M, Acosta H, Mirus J, Barry N, et al: Restoration of Wnt-7a expression reverses non-small cell lung cancer cellular transformation through frizzled-9-mediated growth inhibition and promotion of cell differentiation. J Biol Chem 280: 19625-19634, 2005.

18. Kim R, Ohi Y, Inoue H and Toge T: Enhancement of chemotherapeutic agents induced-apoptosis associated with activation of c-Jun N-terminal kinase 1 and caspase 3 (CPP32) in bax-transfected gastric cancer cells. Anticancer Res 20: 439-444, 2000.

19. Levresse V, Marek L, Blumberg D and Heasley LE: Regulation of platinum-compound cytotoxicity by the c-Jun N-terminal kinase and c-Jun signaling pathway in small-cell lung cancer cells. Mol Pharmacol 62: 689-697, 2002.

20. Vadlapatla RK, Vadlapudi AD, Pal D and Mitra AK: Mechanisms of drug resistance in cancer chemotherapy: Coordinated role and regulation of efflux transporters and metabolizing enzymes. Curr Pharm Des 19: 7126-7140, 2013.

21. Lin L, Deng W, Tian Y, Chen W, Wang J, Fu L, Shi D, Zhao M and Luo W: Lasiodin inhibits proliferation of human nasopharyngeal carcinoma cells by simultaneous modulation of the Apaf-1/caspase, AKT/MAPK and COX-2/NF- $\kappa$ B signaling pathways. PLoS One 9: e97799, 2014.

22. Chou J, Lin YC, Kim J, You L, Xu Z, He B and Jablons DM: Nasopharyngeal carcinoma-review of the molecular mechanisms of tumorigenesis. Head Neck 30: 946-963, 2008.

23. Kyriakis JM and Avruch J: Mammalian mitogen-activated protein kinase signal transduction pathways activated by stress and inflammation. Physiol Rev 81: 807-869, 2001. 